\title{
Extreme Point Characterizations for Infinite Network Flow Problems
}

\author{
H. Edwin Romeijn \\ Department of Industrial and Systems Engineering, University of Florida, \\ 303 Weil Hall, P.O. Box 116595, Gainesville, Florida 32611-6595
}

\author{
Dushyant Sharma, Robert L. Smith \\ Department of Industrial and Operations Engineering, The University of Michigan, \\ Ann Arbor, Michigan 48109-2117
}

\begin{abstract}
We study capacitated network flow problems with demands defined on a countably infinite collection of nodes having finite degree. This class of network flow models includes, for example, all infinite horizon deterministic dynamic programs with finite action sets, because these are equivalent to the problem of finding a shortest path in an infinite directed network. We derive necessary and sufficient conditions for flows to be extreme points of the set of feasible flows. Under an additional regularity condition met by all such problems with integer data, we show that a feasible solution is an extreme point if and only if it contains neither a cycle nor a doubly-infinite path consisting of free arcs (an arc is free if its flow is strictly between its upper and lower bounds). We employ this result to show that the extreme points can be characterized by specifying a basis. Moreover, we establish the integrality of extreme point flows whenever node demands and arc capacities are integer valued. We illustrate our results with an application to an infinite horizon economic lot-sizing problem. () 2006 Wiley Periodicals, Inc. NETWORKS, Vol. 48(4), 209-222 2006
\end{abstract}

Keywords: infinite horizon optimization; infinite trees; extreme point solutions; basic solutions

\section{INTRODUCTION}

An important class of optimization problems in operations research is formed by planning problems over time. Many of these planning problems can quite naturally be formulated as network flow problems. When, as is often the case, there is no

Received November 2005; accepted July 2006

Correspondence to: H. E. Romeijn; e-mail: romeijn@ise.ufl.edu

Contract grant sponsor: NSF; contract grant numbers: DMI-0322114 and DMI-9713723.

DOI 10.1002/net.20134

Published online in Wiley InterScience (www.interscience.wiley. com).

C) 2006 Wiley Periodicals, Inc. natural study horizon that can be specified a priori, these planning problems are formulated as problems over an infinite horizon. Some notable examples of such problems include capacity expansion under nonlinear demand [7], equipment replacement under technological change [5,12], and production planning under nonstationary cost and demand data [15]. Such problems then give rise to minimum cost network flow problems in networks with infinitely many nodes and arcs. The usual attack in solving such an infinite horizon problem is through a planning horizon approach that seeks solutions to finite horizon versions that arbitrarily well approximate optimal solutions to the original infinite horizon problem (see, e.g., $[6,14])$. Our intent in this article, however, is to study properties, and in particular extreme point properties, of the infinite horizon versions of such problems, including more broadly, general infinite network flow problems.

Network flow problems form a very well-studied area. The excellent book by Ahuja, et al. [1] describes the state of the art in designing algorithms for various types of network flow problems. To date, however, attention has almost exclusively been focused on network flow problems in a network containing only a finite number of nodes and arcs (notable exceptions being the works by Anderson and Philpott [4], Fuchssteiner and Morisse [10], and Gomory and $\mathrm{Hu}$ [11], who studied network flow problems in networks with an uncountable number of nodes). Many optimization algorithms for finite network flow problems, in particular for linear and concave minimum cost network flow problems, employ a characterization of extreme points and basic solutions of such problems using spanning trees. Although the theory of optimization problems in infinite dimensional spaces is quite well developed (see, e.g., [3]), application of this theory for developing algorithms for concrete problems and obtaining important insights into the behavior of extreme point and basic and optimal solutions has been thwarted by the mathematical pathologies inherent in infinite dimensional 
optimization problems. Although Anderson and Nash [3] provided a characterization of extreme point solutions for general infinite linear programs, the abstract nature of their results prevented their further exploitation. Cross et al. [8] attempted to circumvent this problem by indirectly characterizing extreme points of infinite dimensional convex sets by showing they are arbitrarily well approximated by the extreme points of their finite dimensional projections.

In this article, we return to the challenging problem of directly characterizing extreme points within the infinite dimensional space itself, and in particular, through basic variables. More precisely, the lack of and need for concrete characterizations of extreme points and basic solutions to infinite network flow problems motivated our work. Our structural results will provide an essential building block towards the characterization of extreme points for general doubly-infinite linear programming problems as well as toward the development of a (network) simplex method for infinite dimensional linear programming problems.

We will study capacitated network flow problems with demands defined on a countably infinite collection of nodes having finite degree. This class of network flow models includes, for example, all infinite horizon deterministic dynamic programs with finite action sets because these are equivalent to the problem of finding a shortest infinite path in an infinite directed network. We extend concepts and structural properties of solutions to network flow problems from the finite to the infinite case. We derive properties of the set of all feasible solutions (i.e., flows that satisfy all flow balance and bound constraints), and establish a relationship between extreme point solutions to the network flow problem and trees in the network, generalizing an analogous property of the finite version of the problem. In particular, we provide necessary and sufficient conditions for a feasible solution to be an extreme point. Under an additional nonvanishing support assumption on arc flows in extreme point solutions we show that all extreme point solutions can be uniquely characterized via a specification of a set of free variables. This then enables us to characterize extreme points through a decomposition of the flow variables into basic and nonbasic variables. We find that, in infinite networks with integral node demands and arc capacities, all extreme points have integral flows. This not only generalizes the analogous result from the finite network case, but also guarantees that the nonvanishing support assumption is met. As an example, we study a capacitated economic lot-sizing problem with concave costs, and extend the finite horizon characterization of all (including the optimal) extreme point solutions to such problems.

This article is organized as follows. In Section 2 we introduce the necessary notation for the network flow problem, and extend some concepts from finite graph theory to the infinite case. In Section 3 we derive structural properties of feasible flows as well as flows corresponding to extreme points of the set of all feasible flows. In Section 4 we study a production planning problem over an infinite horizon with concave costs. We end in Section 5 with some concluding remarks and directions for future research.

\section{NOTATION AND DEFINITIONS}

In this section, we introduce the notation and some basic definitions used throughout the article. Although most of the definitions introduced here are commonly used for finite networks, some definitions are extended or introduced to deal specifically with infinite networks.

\subsection{Network Definitions}

Let $G=(N, A)$ be a directed network consisting of a countable set $N=\{1,2,3, \ldots\}$ of nodes and a set $A \subseteq N \times N$ of arcs. Let $d \in \mathbb{R}^{|N|}$ with typical element $d(i)$ represent the demand for each node, that is, $d(i)$ is the demand associated with node $i \in N$ (where we note that, if $d(i)<0,-d(i)>0$ is actually a supply). We assume that the in and out degree of each node $i \in N$ is finite, that is, $|\{j \in N:(j, i) \in A\}|<\infty$ and $|\{j \in N:(i, j) \in A\}|<\infty$. Let $\ell, u \in \mathbb{R}^{|A|} \cup\{-\infty, \infty\}$ with typical elements $\ell(i, j)$ and $u(i, j)$ be the vectors of lower and upper bounds, respectively, for the flows on $\operatorname{arcs}$ in $A$. Without loss of generality, we assume that $\ell(i, j)<u(i, j)$ for all $(i, j) \in A$.

We define a path $P$ in the graph $G$ to be a collection of arcs $P \subseteq A$ in $G$ representing a sequence of nodes $i_{1}-i_{2}-i_{3}-\ldots$ such that no node is repeated in the sequence, and for each $k$, either $\left(i_{k}, i_{k+1}\right) \in P$ or $\left(i_{k+1}, i_{k}\right) \in P$. If the path contains finitely many arcs (and nodes) then it is called a finite path. Two infinite paths that share their endpoints define what we will call a doubly-infinite path. A cycle $C$ in the graph $G$ is defined as a finite path with corresponding sequence of nodes $i_{1}-i_{2}-i_{3}-\cdots-i_{k}$ whose endpoints are connected by an additional arc $\left(i_{k}, i_{1}\right)$ or $\left(i_{1}, i_{k}\right)$. Given a cycle $C$ (or a path $P$ ), we shall use $A(C)$ (or $A(P)$ to denote the set of arcs in the cycle (or path). We say that two nodes $i, j \in N$ are connected if there exists a finite path $i_{1}-i_{2}-\cdots-i_{k}$ in $G$ with $i_{1}=i$ and $i_{k}=j$. The graph $G$ is called connected if every pair of nodes in $G$ is connected. In this article, we shall assume that the graph $G$ is connected.

A subgraph $T=\left(N^{\prime}, A^{\prime}\right)$ of $G$ is called a tree if $T$ is connected and it does not contain any cycles. Note that there must be a unique finite path connecting each pair of nodes in $T$. We can designate some node $r \in N^{\prime}$ to be the root of $T$. In this case, the tree $T$ is called a rooted tree, and is denoted by $T=\left(N^{\prime}, A^{\prime}, r\right)$. The designation of a node as a root node allows us to associate additional properties with the nodes in a tree. If $(j, i)$ or $(i, j)$ is the arc on the unique path connecting node $i$ and node $r$ then $j$ is called the parent of $i$, denoted by $p_{T}(i)$, and $i$ is called a child of $j$. For a node $i \in N^{\prime}$, we define $C_{T}(i)$ as the set of children of $i$. The set $N_{T}(i)$ of descendants of a node $i \in T$ consists of node $i$, its children, children of its children, and so on. We say that node $i$ is a leaf node if it is the only descendant of itself. We define the subtree $T(i)=\left(N_{T}(i), A_{T}(i), i\right)$ rooted at a node $i \in N^{\prime}$ to be the subgraph of $T$ that consists of the descendants of node $i$ in $T$. We say that a set of $\operatorname{arcs} S \subseteq A^{\prime}$ is a finite cut in the rooted tree $T$ if the number of nodes $i \in N^{\prime}$ whose unique path from the root node $r$ does not contain any arc from $S$ 


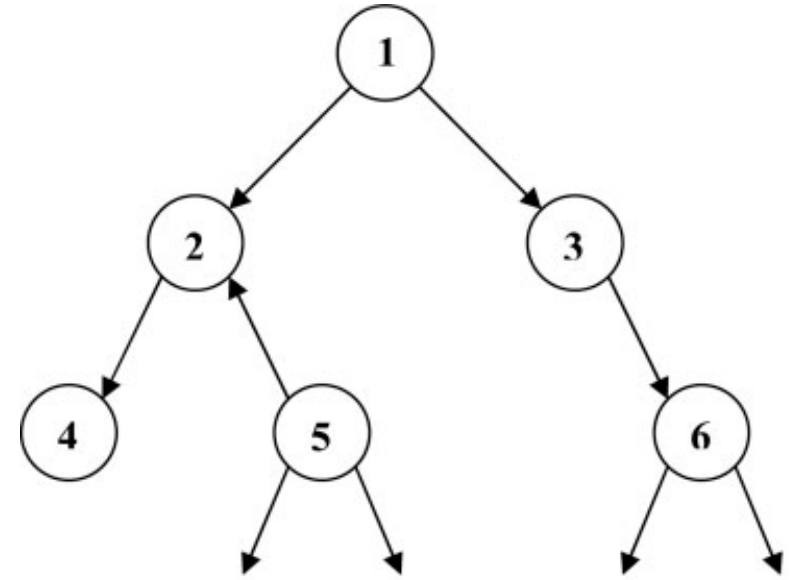

(a)

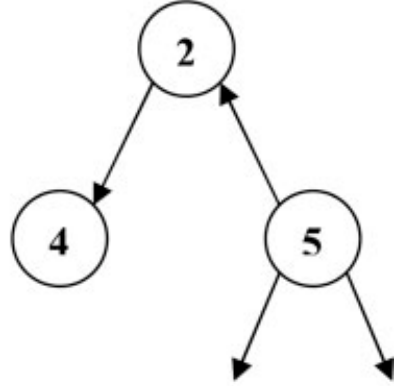

(b)

FIG. 1. (a) A tree $T$, and (b) its subtree $T(2)$.

is finite. In Figure 1 we illustrate a tree and a subtree. The arcs $S=\{(5,2),(3,6)\}$ form a finite cut, whereas the arcs $\{(2,4),(3,6)\}$ do not form a finite cut. We denote the set of finite cuts in a rooted tree $T$ by $\mathbf{S}(T)$.

\subsection{Feasible Flows and Extreme Points}

A vector $x \in \mathbb{R}^{|A|}$ with typical element $x(i, j)$ is called a feasible flow if for each node $i \in N$, the flow balance constraint

$$
\sum_{j \in N:(j, i) \in A} x(j, i)-\sum_{j \in N:(i, j) \in A} x(i, j)=d(i)
$$

is satisfied, and $\ell(i, j) \leq x(i, j) \leq u(i, j)$ for all $(i, j) \in A$.

Given a feasible flow $x$, we define $r(i, j)=\min \{x(i, j)-$ $\ell(i, j), u(i, j)-x(i, j)\}$ as the maximum amount by which the flow on $(i, j)$ can be increased or decreased without violating bounds. We define the free arc graph of $x$ as the graph $G(x)=$ $(N, A(x))$, where $A(x)=\{(i, j) \in A: r(i, j)>0\}$ is the set of free arcs, that is, arcs whose flow can be increased as well as decreased. We define the set of arcs with their flow equal to their upper bound as $U(x)=\{(i, j) \in A: x(i, j)=u(i, j)\}$ and arcs with flow equal to their lower bound as $L(x)=\{(i, j) \in$ $A: x(i, j)=\ell(i, j)\}$. We note that $A(x), U(x)$, and $L(x)$ are pairwise disjoint and $A(x) \cup U(x) \cup L(x)=A$. The following proposition provides some important properties of feasible flows that will be useful later in this article.

Proposition 2.1. Let $x^{1}$ and $x^{2}$ be two feasible flows and $x=\left(x^{1}+x^{2}\right) / 2$. Then

(a) $A\left(x^{1}\right) \cup A\left(x^{2}\right) \subseteq A(x)$.

(b) $L(x)=L\left(x^{1}\right) \cap L\left(x^{2}\right)$.

(c) $U(x)=U\left(x^{1}\right) \cap U\left(x^{2}\right)$.

Proof. The first result follows by noting that for any arc $(i, j) \in A$ if $\ell(i, j)<x^{1}(i, j)<u(i, j)$ or $\ell(i, j)<x^{2}(i, j)<$ $u(i, j)$ then $\ell(i, j)<x(i, j)<u(i, j)$. The second and third results follow by the observation that $x(i, j)=\ell(i, j) \Longleftrightarrow$ $x^{1}(i, j)=x^{2}(i, j)=\ell(i, j)$, and $x(i, j)=u(i, j) \Longleftrightarrow$ $x^{1}(i, j)=x^{2}(i, j)=u(i, j)$.

Finally, a feasible flow $x$ is an extreme point of the set of feasible flows if there do not exist feasible flows $x^{1}$ and $x^{2}$ such that $x^{1} \neq x^{2}$ and $x=\left(x^{1}+x^{2}\right) / 2$.

\section{EXTREME POINT FLOWS}

In this section we develop necessary and sufficient conditions for a feasible flow $x$ in a graph $G$ to be an extreme point. However, we first address the issue of the existence of an extreme point in an infinite network flow problem.

Theorem 3.1. Suppose $G$ is a network with demands $d$ and finite lower and upper bounds $\ell, u \in \mathbb{R}^{|A|}$. If the network flow problem is feasible then an extreme point solution exists.

Proof. The set of solutions of the network flow problem is clearly convex. Further, because the value of each arc flow is bounded, the solution space is also compact with respect to the product topology. The Krein-Milman theorem (see [2]) then implies that, if the solution space is nonempty, it contains an extreme point.

Note that all results in this article continue to hold if some or all of the flow bounds are infinite. However, in that case, the set of extreme points may be empty.

\subsection{Why the Finite Extreme Point Characterization Fails for Infinite Networks}

Given the result of Theorem 3.1, there always exists an extreme point solution whenever the problem is feasible. The following necessary condition for a flow to be an extreme point follows directly using a similar argument as for network flow problems over finite networks.

Lemma 3.2. If a feasible flow $x$ is an extreme point then $G(x)$ contains no cycles. 


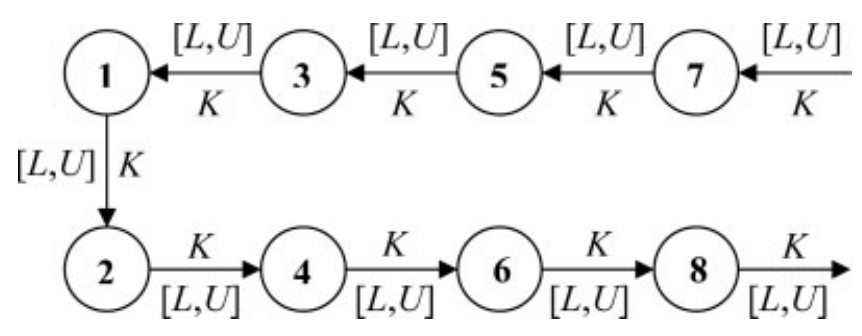

FIG. 2. A network with no cycles.

We note that for network flow problems over finite networks the converse of Lemma 3.2 also holds: that is, if $G(x)$ contains no cycle then $x$ is an extreme point. Another way of putting this is that, in the case of finite networks, an extreme point solution can always be specified by providing a set of restricted arcs, that is, arcs in the network whose flow is restricted to be at their lower or upper bound. Such arcs are called restricted arcs at lower bound and restricted arcs at upper bound, respectively. The flows on all remaining arcs, that is, all so-called free arcs in the free arc graph $G(x)$, are uniquely determined by the flows on the restricted arcs. However, for networks with an infinite number of nodes the converse of Lemma 3.2 does not hold. We will illustrate this with three examples that show that extreme points in infinite networks cannot in general be characterized by specifying an acyclic subgraph.

Example 1. Our first example is of an infinite network that basically consists of a pair of infinite paths connected at one of the nodes. In particular, consider the infinite graph $G=(N, A)$ where $N=\{1,2, \ldots\}$ and $A=\{(2 i+1,2 i-$ 1) $: i=1,2, \ldots\} \cup\{(1,2)\} \cup\{(2 i, 2 i+2): i=1,2, \ldots\}$. This network does not contain any cycles. Let all nodes be transshipment nodes, that is, the demands are $d(i)=0$ for all $i \in N$. Moreover, let the lower and upper bounds on all arcs be $\ell(i, j)=L$ and $u(i, j)=U$ for all $(i, j) \in A$, where $L<U$ are finite numbers. The network is illustrated in Figure 2. Note that, for any constant $K$ such that $L<K<U$, the constant flow given by $x(i, j)=K$ for all $(i, j) \in A$ is a feasible flow with $G(x)=G$. However, the flow $x$ is not an extreme point flow because it can be written as $x=\left(x^{1}+x^{2}\right) / 2$, where $x^{1}$ and $x^{2}$ are constant flows with values $K-\Delta$ and $K+\Delta$, respectively, for $\Delta=\min \{K-L, U-K\}$. The only two extreme points of this network flow problem are given by constant flows of $L$ or $U$.

Example 2. Our second example considers an infinite graph $G=(N, A)$ that is similar to the graph in Example 1 . The node set is again $N=\{1,2, \ldots\}$, but the arc set is now chosen to be $A=\{(2 i-1,2 i+1): i=1,2, \ldots\} \cup\{(1,2)\} \cup$ $\{(2 i, 2 i+2): i=1,2, \ldots\}$. This network does not contain any cycles. We choose the demands to be given by: $d(1)=-2$, and $d(2 i)=d(2 i+1)=1 / 2^{i+1}$ for $i \in\{1,2, \ldots\}$. The lower and upper bounds on each arc are set to 0 and 2 , respectively. The network is shown in Figure 3. By construction, any feasible flow $x$ would satisfy $0<x(i, j) \leq 3 / 2$ for all $(i, j) \in A$. Hence, for any feasible flow $x$ we have $G(x)=G$. However, there are only two feasible solutions that are extreme points. The first solution is given by $x(2 i-1,2 i+1)=1+1 / 2^{i}$ for $i \in\{1,2, \ldots\}, x(1,2)=1 / 2$, and $x(2 i, 2 i+2)=1 / 2^{i+1}$ for $i \in\{1,2, \ldots\}$. The second extreme point is given by $x(2 i-1,2 i+1)=1 / 2^{i}$ for $i \in\{1,2, \ldots\}, x(1,2)=3 / 2$, and $x(2 i, 2 i+2)=1+1 / 2^{i+1}$ for $i \in\{1,2, \ldots\}$. Note that the total demand of all demand nodes is equal to 1 , whereas the supply of the only supply node is equal to 2 . Therefore, there is one unit of flow that is shipped from the supply node for which no demand exists. An extreme point in the network corresponds to a solution in which the excess unit supplied flows through either the infinite path $1-3-5-7-\cdots$ or the infinite path $1-2-4-6-\cdots$. Any feasible flow in which the excess unit supplied is shared by these two infinite paths is not an extreme point.

Example 3. Our third example involves an infinite binary tree (see Fig. 4). In this network, we assume that the root node has a supply of 1 unit [i.e., $d(1)=-1$ ], and all other nodes are transshipment nodes $(d(i)=0$ for all $i \in N \backslash\{1\})$. Furthermore, let the lower and upper bounds on the arc flows be 0 and 2 units, respectively. Figure 4 shows a feasible solution with positive flow on all arcs in the network. Moreover, it is clear that the free arc graph corresponding to any feasible flow does not contain any cycles. However, the only extreme points in this network flow problem are feasible flows that

(a)

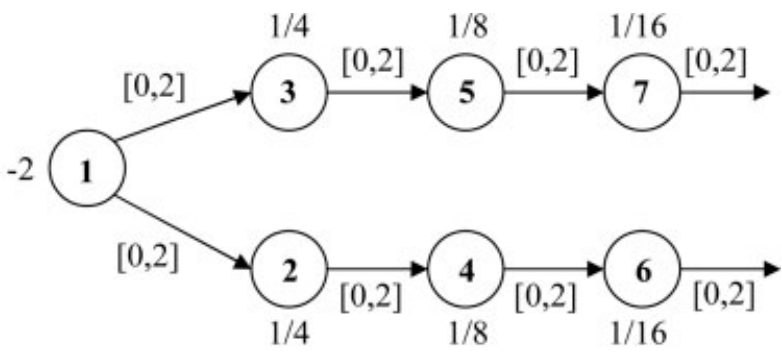

(b)

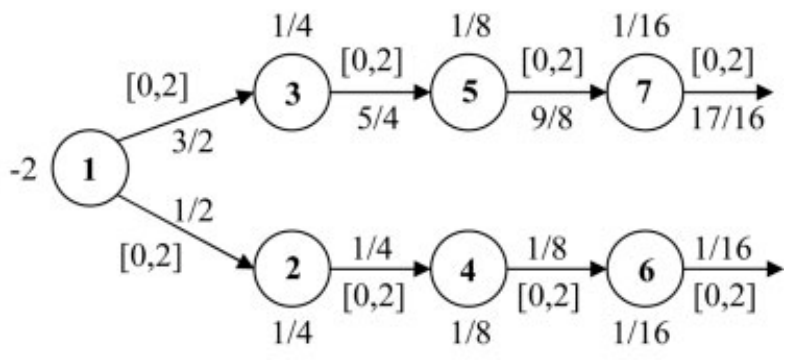

(c)

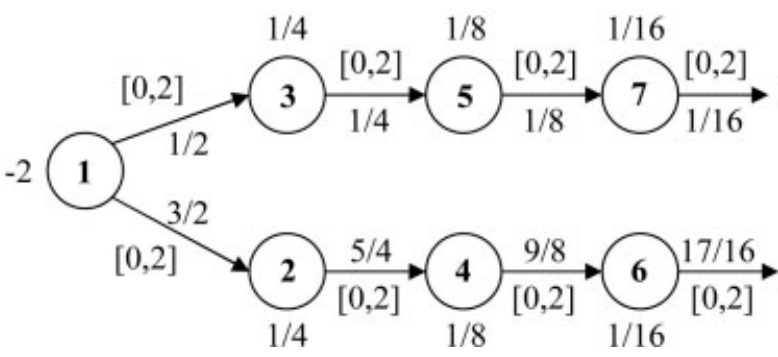

FIG. 3. (a) Example network flow problem; (b) first extreme point; (c) second extreme point. 


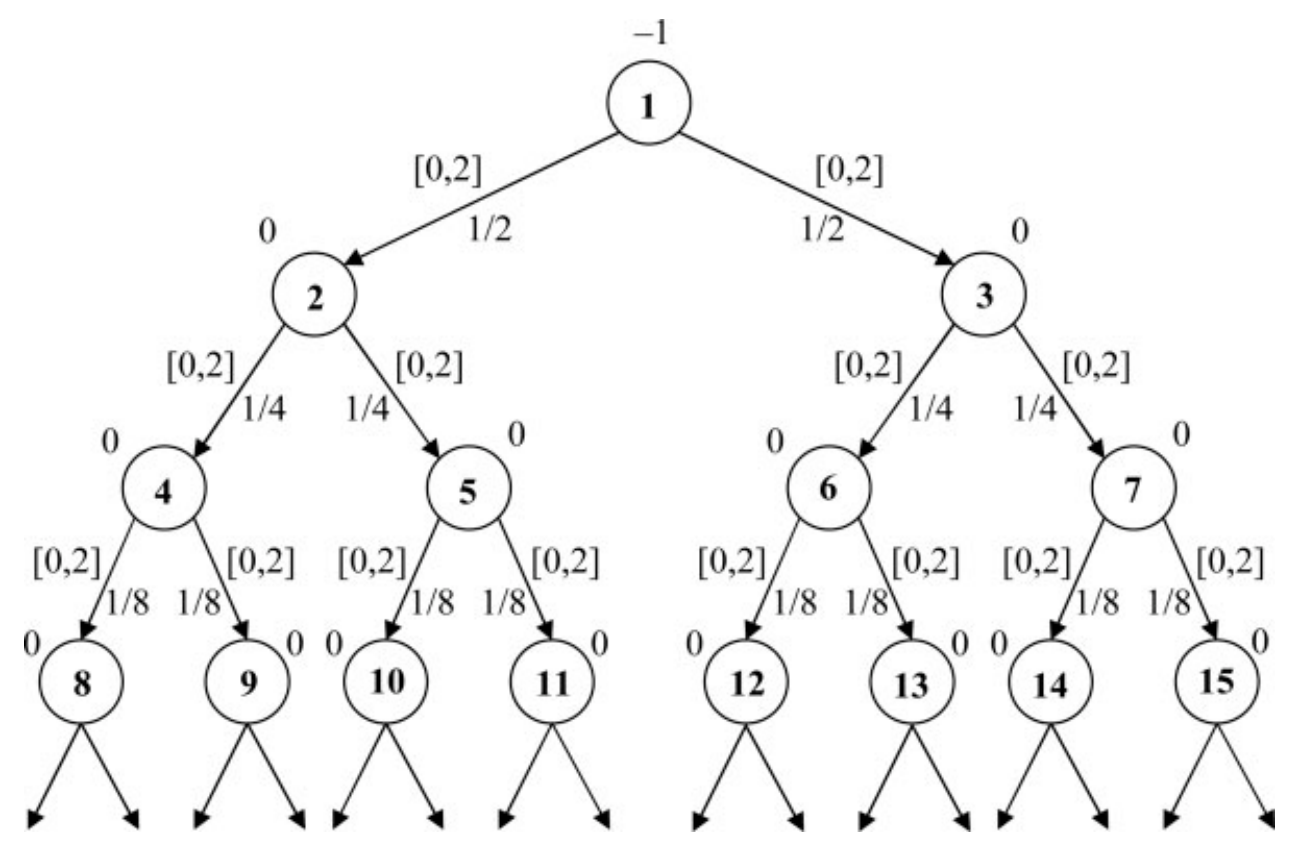

FIG. 4. An infinite binary tree.

carry a single unit along a single infinite path from the root node 1 .

All three examples show that extreme points in infinite networks cannot in general be characterized by specifying an acyclic subgraph. The main cause of this failure to generalize the characterization of extreme points in network flow problems from finite to infinite networks is that a given acyclic free arc graph (together with flows on the restricted arcs) uniquely determines the flows on the free arcs in the finite case, but does not uniquely determine these flows in the infinite case. Hence, an extreme point is not solely determined by specifying the free and restricted arcs in the case of infinite networks. In the remainder of this section, we will provide a set of necessary and sufficient conditions for a given feasible flow to be an extreme point in an infinite network flow problem.

\subsection{Characterizing Extreme Points in Infinite Networks}

From Lemma 3.2 it follows that if $x$ is an extreme point then any connected subgraph of $G(x)$ must be a tree. We note that with each node $i \in N$ we can associate a maximal tree $T=\left(N^{\prime}, A^{\prime}\right)$ in the graph $G(x)$ given by the node set $N^{\prime}=\left\{i^{\prime} \in N: i\right.$ and $i^{\prime}$ are connected in $\left.G(x)\right\}$. It can be easily shown that the maximal trees associated with any pair of nodes $i, j \in N$ that are connected are identical. Therefore, the graph $G(x)$ consists of one or more (and possibly countably infinitely many) maximal trees such that there are no arcs in $A(x)$ connecting two different maximal trees. Given a maximal tree $T=\left(N^{\prime}, A^{\prime}, r\right)$ in $G(x)$ (rooted at a node $\left.r \in N^{\prime}\right)$ we define, for every node $i \in N^{\prime}$,

$$
R_{T}(i)=\inf _{S \in \mathbf{S}(T(i))} \sum_{\left(i^{\prime}, j^{\prime}\right) \in S} r\left(i^{\prime}, j^{\prime}\right) .
$$

The value $R_{T}(i)$ provides a measure of the maximum amount by which the flow on the arc connecting node $i$ and its parent $p_{T}(i)$ in $T$ could be changed when we are only allowed to balance that change by modifying arc flows in the subtree $T(i)$. Note that if $T(i)$ is a finite tree, the empty cut is a valid finite cut, and we immediately obtain $R_{T}(i)=0$. Intuitively, this can be seen by observing that the flow balance constraints prohibit changing the flow on any given arc when we may only balance that change using changes in the subtree $T(i)$. The following lemma provides a key property of the functions $R_{T}(i)$.

Lemma 3.3. Given a rooted tree $T=\left(N^{\prime}, A^{\prime}, r\right)$, the function $R_{T}(i)$ for $i \in N^{\prime}$ is given by

$$
R_{T}(i)=\sum_{j \in C_{T}(i)} \min \left\{\alpha_{i j}, R_{T}(j)\right\}
$$

where

$$
\alpha_{i j}= \begin{cases}r(i, j) & \text { if }(i, j) \in A^{\prime} \\ r(j, i) & \text { otherwise }\end{cases}
$$

Proof. See the Appendix.

We will next illustrate the measure $R_{T}(i)$ for infinite networks through the three examples presented in Section 3.1.

Example 1. Consider a solution of the form $x(i, j)=K$ for all $(i, j) \in A$ where $L<K<U$, so that $G(x)=G$. Thus, $G(x)$ contains a single maximal tree: $G(x)$ itself. If we consider this tree, say $T$, rooted at node 1 , we obtain $R_{T}(i)=\Delta$ for $i \in N \backslash\{1\}$ and $R_{T}(1)=2 \Delta$, where $\Delta=$ $\min (K-L, U-K)$. Because node 1 has two children in $T$ with positive $R_{T}(i)$ we can reroute flow by the amount $\Delta$ in the tree $T$ in two directions. Therefore, the solution $x$ is not an extreme point. 
Example 2. In this example, any feasible flow has $G(x)=$ $G$, which in turn, is equal to the single maximal tree in $G(x)$. We consider this tree, say $T$, rooted at node 1 . Now consider the midpoint of the two solutions illustrated in Figure 3(b) and (c): $x(2 i-1,2 i+1)=1 / 2+1 / 2^{i}$ for $i \in\{1,2, \ldots\}, x(1,2)=$ 1 , and $x(2 i, 2 i+2)=1 / 2+1 / 2^{i+1}$ for $i \in\{1,2, \ldots\}$. For this solution, we have $R_{T}(2 i)=R_{T}(2 i+1)=1 / 2$ for $i \in$ $\{1,2, \ldots\}$ and $R_{T}(1)=1$. Because node 1 has two children in $T$ with positive $R_{T}(i)$, we can reroute flow in the tree $T$ by $1 / 2$ in two directions, and thus the solution $x$ is not an extreme point. However, for the solution in Figure 3(b) we have $R_{T}(2 i+1)=1$ for $i \in\{1,2, \ldots\}, R_{T}(2 i)=0$ for $i \in\{1,2, \ldots\}$, and $R_{T}(1)=1$. In this case, each node has at most a single child in $T$ with positive $R_{T}(i)$ so that we cannot reroute flow by any positive amount in the tree $T$ so the solution $x$ is an extreme point.

Example 3. Consider the feasible flow, say $x$, illustrated in Figure 4. For this flow we have $G(x)=G$, which in turn, is equal to the single maximal tree in $G(x)$. We consider this tree, say $T$, rooted at node 1 . Then $R_{T}(i)=1 / 2$ for $i \in\{2,3\}$ and $R_{T}(1)=1$. Because node 1 has two children in $T$ with positive $R_{T}(i)$, we can reroute flow in the tree $T$ by $1 / 2$ in two directions and the solution $x$ is not an extreme point. It is easy to see that any feasible flow except a flow that sends one unit along a single infinite directed path from node 1 is not an extreme point either.

The definition of $R_{T}(i)$ and the three examples now motivate the following condition which, as we will show in the remainder of this section, is necessary and sufficient for a feasible flow $x$ in $G$ to be an extreme point.

\section{Condition 3.4.}

(a) $G(x)$ contains no cycles.

(b) Every maximal tree $T=\left(N^{\prime}, A^{\prime}\right)$ in $G(x)$ can be rooted at some node $r \in N^{\prime}$ such that any node $i \in N^{\prime}$ with $R_{T}(i)>0$ has at most one child $j \in C_{T}(i)$ such that $R_{T}(j)>0$.

We will refer to this condition as Extreme Point Condition 3.4. Although Extreme Point Condition 3.4(b) requires the existence of some root node with the desired property for each maximal tree, it is, in fact, equivalent to the (seemingly more restrictive and sometimes more convenient) condition that the desired property holds for all choices of root node, as is formally proven in Lemma A.1 in the Appendix. That is, we can replace Extreme Point Condition 3.4(b) by

$\left(b^{\prime}\right)$ In every maximal tree $T=\left(N^{\prime}, A^{\prime}, r\right)$ in $G(x)$ rooted at any node $r \in N^{\prime}$, any node $i \in N^{\prime}$ with $R_{T}(i)>0$ has at most one child $j \in C_{T}(i)$ such that $R_{T}(j)>0$.

We first establish the sufficiency of Extreme Point Condition 3.4 for a feasible flow $x$ to be an extreme point.

Proposition 3.5. A feasible flow $x$ is an extreme point if it satisfies Extreme Point Condition 3.4.

Proof. Suppose that the feasible flow $x$ satisfies Extreme Point Condition 3.4 but it is not an extreme point. Let $x^{1}$ and $x^{2}$ be feasible flows such that $x=\left(x^{1}+x^{2}\right) / 2$ and $x^{1} \neq x^{2}$. Using Proposition 2.1, it follows that $L(x)=L\left(x^{1}\right) \cap L\left(x^{2}\right)$ and $U(x)=U\left(x^{1}\right) \cap U\left(x^{2}\right)$. Therefore, $x^{1}$ and $x^{2}$ can only differ over the arcs in the set $A(x)$. Because $G(x)$ contains no cycles by Extreme Point Condition 3.4(a), every arc in $A(x)$ must belong to a maximal tree in $G(x)$. Let $(i, j)$ be an arc in some maximal tree $T=\left(N^{\prime}, A^{\prime}\right)$ in $G(x)$ such that $x^{1}(i, j) \neq$ $x^{2}(i, j)$. Because $x, x^{1}$, and $x^{2}$ are feasible flows, they each satisfy the flow balance constraint (1) at $i$. Therefore, one of the following two situations must happen (because otherwise the flow balance at $i$ would not be satisfied):

(1) there is an $\operatorname{arc}(i, \bar{j}) \in A^{\prime}$ such that $\bar{J} \neq j$ and $x^{1}(i, \bar{j}) \neq$ $x^{2}(i, \bar{j})$.

(2) there is an $\operatorname{arc}(\bar{j}, i) \in A^{\prime}$ such that $\bar{j} \neq j$ and $x^{1}(\bar{j}, i) \neq$ $x^{2}(\bar{j}, i)$.

Consider the maximal tree $T$ rooted at node $i$, that is, $T=$ $\left(N^{\prime}, A^{\prime}, i\right)$. By the observations above, $j, \bar{j} \in C_{T}(i)$ when $i$ is the root node. If $R_{T}(i)=0$, then by Lemma 3.3 it follows that $R_{T}(j)=R_{T}(\bar{j})=0$. If $R_{T}(i)>0$ then either $R_{T}(j)=0$ or $R_{T}(\bar{j})=0$ by Extreme Point Condition 3.4( $\left.\mathrm{b}^{\prime}\right)$ [which must be satisfied because it is equivalent to Extreme Point Condition 3.4(b)].

Without loss of generality, let $R_{T}(j)=0$ and $\Delta>0$ be such that $x^{1}(i, j)=x(i, j)-\Delta$ [and thus $x^{2}(i, j)=x(i, j)+\Delta$ ]. Because $R_{T}(j)=0$, by definition there must exist a finite cut $S \in \mathbf{S}(T(j))$ such that $\sum_{\left(i^{\prime}, j^{\prime}\right) \in S} r\left(i^{\prime}, j^{\prime}\right)<\Delta$. Using Lemma A.2 from the Appendix with flows $x$ and $x^{1}$ over the finite cut $S$, we obtain

$$
\Delta=\left|x(i, j)-x^{1}(i, j)\right| \leq \sum_{\left(i^{\prime} j^{\prime}\right) \in S}\left|x\left(i^{\prime}, j^{\prime}\right)-x^{1}\left(i^{\prime}, j^{\prime}\right)\right| .
$$

We note that for any arc $\left(i^{\prime}, j^{\prime}\right) \in S$

$$
x\left(i^{\prime}, j^{\prime}\right)-x^{1}\left(i^{\prime}, j^{\prime}\right)=x^{2}\left(i^{\prime}, j^{\prime}\right)-x(i, j) .
$$

Using $\ell\left(i^{\prime}, j^{\prime}\right) \leq x^{1}\left(i^{\prime}, j^{\prime}\right) \leq u\left(i^{\prime}, j^{\prime}\right)$ and $\ell\left(i^{\prime}, j^{\prime}\right) \leq x^{2}\left(i^{\prime}, j^{\prime}\right) \leq$ $u\left(i^{\prime}, j^{\prime}\right)$, it follows that

$$
\begin{aligned}
& \left|x\left(i^{\prime}, j^{\prime}\right)-x^{1}\left(i^{\prime}, j^{\prime}\right)\right| \leq \min \left\{x\left(i^{\prime}, j^{\prime}\right)-\ell\left(i^{\prime}, j^{\prime}\right),\right. \\
& \left.\times u\left(i^{\prime}, j^{\prime}\right)-x\left(i^{\prime}, j^{\prime}\right)\right\}=r\left(i^{\prime}, j^{\prime}\right)
\end{aligned}
$$

which implies that

$$
\Delta \leq \sum_{\left(i^{\prime}, j^{\prime}\right) \in S} r\left(i^{\prime}, j^{\prime}\right) .
$$

However, this contradicts the choice of $S$ to be such that $\sum_{\left(i^{\prime}, j^{\prime}\right) \in S} r\left(i^{\prime}, j^{\prime}\right)<\Delta$. Therefore, our assumption that $x^{1}(i, j) \neq x^{2}(i, j)$ must be incorrect. Because the arc $(i, j)$ was chosen arbitrarily in $A(x)$, it follows that $x^{1}$ and $x^{2}$ are identical on the set $A(x)$, and we conclude that $x$ is an extreme point.

We next show that Extreme Point Condition 3.4 is necessary for a feasible flow to be an extreme point. 
Proposition 3.6. If a feasible flow $x$ is an extreme point then it satisfies Extreme Point Condition 3.4.

Proof. Let $x$ be a feasible flow, and suppose that it is an extreme point. From Lemma 3.2, it follows that $G(x)$ contains no cycles. Suppose that there exists a maximal rooted tree $T=\left(N^{\prime}, A^{\prime}, r\right)$ in $G(x)$ such that there is a node $i \in N^{\prime}$ with two children $j, \bar{j} \in C_{T}(i)$ and $R_{T}(i), R_{T}(j), R_{T}(\bar{J})>0$. We shall construct two feasible flows $x^{1}$ and $x^{2}$ such that $x^{1} \neq x^{2}$ and $x=\left(x^{1}+x^{2}\right) / 2$, providing a contradiction to the assumption that $x$ is an extreme point. In particular, we will let the flows $x^{1}$ and $x^{2}$ be identical to $x$ on all arcs except the arcs between node $i$ and nodes $j, \bar{J}$, and the $\operatorname{arcs}$ in sets $A_{T}(j)$ and $A_{T}(\bar{J})$. For any of these remaining arcs, say $\left(i^{\prime}, j^{\prime}\right)$, we set the value of the flow $x^{2}\left(i^{\prime}, j^{\prime}\right)$ equal to $2 x\left(i^{\prime}, j^{\prime}\right)-x^{1}\left(i^{\prime}, j^{\prime}\right)$, so it remains to specify the flow $x^{1}$. For simplicity of the argument, we will show the construction when $(i, j),(i, \bar{j}) \in A^{\prime}$; the other cases can be handled in a similar manner. Let

$$
\Delta=\min \left\{r(i, j), r(i, \bar{\jmath}), R_{T}(j), R_{T}(\bar{\jmath})\right\} .
$$

We set

$$
\begin{aligned}
x^{1}(i, j) & =x(i, j)+\Delta \\
x^{1}(i, \bar{J}) & =x(i, \bar{J})-\Delta
\end{aligned}
$$

[and thus $x^{2}(i, j)=x(i, j)-\Delta$ and $x^{2}(i, \bar{J})=x(i, \bar{J})+\Delta$ ]. Clearly, the flow balance constraint (1) at node $i$ and the bounds on the flow on arcs $(i, j)$ and $(i, \bar{j})$ are satisfied for both $x^{1}$ and $x^{2}$. The flow $x^{1}$ on the $\operatorname{arcs}$ in $A_{T}(j)$ will be specified in a recursive manner as follows. By definition, Lemma 3.3 says that

$$
\Delta \leq R_{T}(j)=\sum_{k \in C_{T}(j)} \min \left\{\alpha_{j k}, R_{T}(k)\right\} .
$$

It is easy to see that there exist nonnegative numbers $\gamma(k)$ for $k \in C_{T}(j)$ such that $0 \leq \gamma(k) \leq \min \left\{\alpha_{j k}, R_{T}(k)\right\}$ and $\sum_{k \in C_{T}(j)} \gamma(k)=\Delta$. For each child $k \in C_{T}(j)$ we now set the flow on the arc connecting $j$ with $k$. If $(j, k) \in A^{\prime}$, we set $x^{1}(j, k)=x(j, k)+\gamma(k)$. Otherwise, $(k, j) \in A^{\prime}$ and we set $x^{1}(k, j)=x(k, j)-\gamma(k)$. This assignment ensures that the flow balance at node $j$ as well as the bounds on the arcs between node $j$ and its children are satisfied. The assignment also ensures that the flow $x^{2}$ will satisfy both the flow balance and the bound constraints. Note that the values $\gamma(k)$ satisfy the condition $\gamma(k) \leq R_{T}(k)$, so the method used to determine the flows $x^{1}$ on arcs between $j$ and its children can be recursively applied to find the flows on arcs between its children and their children. Also, note that the recursive application of this procedure yields the flow $x^{1}$ for all $\operatorname{arcs}$ in $A_{T}(j)$ so that the flow balance constraints for the nodes in $N^{j}$ and the bounds on all arcs are satisfied. A similar recursion can be used to determine an appropriate set of flow values $x^{1}$ for the $\arcsin A_{T}(k)$.

The construction shows that if the flow $x$ violates Extreme Point Condition 3.4 then there exist flows $x^{1}$ and $x^{2}$ such that $x^{1} \neq x^{2}$ and $x=\left(x^{1}+x^{2}\right) / 2$.
Combining Propositions 3.5 and 3.6 we obtain the major result of this section, namely that Extreme Point Condition 3.4 is a necessary and sufficient condition for any feasible point $x$ to be an extreme point.

Theorem 3.7. A feasible flow $x$ is an extreme point if and only if it satisfies Extreme Point Condition 3.4.

\subsection{A Class of Problems for Which Extreme Points Can Be Characterized through Free Arcs or Basic Variables}

In this section we will discuss an important class of network flow problems in infinite networks for which the extreme points can be characterized through the free arc graph. In addition, we will show that for this class of problems we can extend the concept of basic and nonbasic variables, and thus the concept of basic solutions, to the network flow problem. Although it seems unlikely that one can develop a simplex like method for general minimum linear cost network flow problems in infinite networks, it may be possible to develop such a method for network flow problems from the class discussed in this section.

3.3.1. Network Flow Problems with Nonvanishing Support. Consider the following extreme point condition that is stronger than Extreme Point Condition 3.4.

\section{Condition 3.8}

(a) $G(x)$ contains no cycles.

(b) For any node $i \in N$ there exists at most one infinite path $i-i_{1}-i_{2}-\cdots$ in $G(x)$.

We will refer to this condition as Extreme Point Condition 3.8. If we informally think of two paths to infinity as forming a doubly-infinite path, then we may rephrase Extreme Point Condition 3.8 as the requirement that $G(x)$ contains neither cycles nor doubly-infinite paths.

We will show in this section that Extreme Point Condition 3.8 is a necessary and sufficient condition for a feasible flow $x$ to be an extreme point if the network flow problem satisfies the following assumption:

Assumption 3.9. For any extreme point $x$ there exists a value $\theta>0$ such that if $\ell(i, j)<x(i, j)<u(i, j)$ for some $(i, j) \in A$ then $\ell(i, j)+\theta \leq x(i, j) \leq u(i, j)-\theta$ or, equivalently, $\inf _{(i, j) \in A(x)} r(i, j)>0$.

We will refer to this assumption as Nonvanishing Support Assumption 3.9. The following theorem provides the main result of this section.

Theorem 3.10. Suppose that the network flow problem given by $G=(N, A), \ell, u$, and d satisfies Nonvanishing Support Assumption 3.9. A feasible flow $x$ is an extreme point if and only if Extreme Point Condition 3.8 is satisfied.

Proof. We prove the theorem by showing that if the network flow problem satisfies Nonvanishing Support Assumption 3.9, then Extreme Point Condition 3.8(b) is equivalent to Extreme Point Condition 3.4( $\left.\mathrm{b}^{\prime}\right)$. 
Suppose that the flow $x$ satisfies conditions (a) and (b) in the theorem. Consider any maximal rooted tree $T=$ $\left(N^{\prime}, A^{\prime}, r\right)$ in $G(x)$. Extreme Point Condition 3.8(b) along with the assumption that any node has finite degree implies that for any node $i \in N^{\prime}$, at most one subtree $T(j)$ rooted at a child $j \in C_{T}(i)$ contains infinitely many nodes, because otherwise there would be two distinct infinite paths from node $i$. By definition, the value $R_{T}(j)$ is zero if $T(j)$ is finite because $S=\varnothing$ is a finite cut of $T(j)$ in this case. Therefore, for any node $i \in N^{\prime}$, at most one child $j \in C_{T}(i)$ can have $R_{T}(j)>0$. In other words, the flow $x$ satisfies Extreme Point Condition 3.4(b).

Now suppose that the flow $x$ satisfies Extreme Point Condition 3.4(b) and thus also Extreme Point Condition 3.4( $\left.\mathrm{b}^{\prime}\right)$. Consider any node $i \in N$. Because $G(x)$ contains no cycle, node $i$ must be part of a maximal tree. Let $T=\left(N^{\prime}, A^{\prime}, i\right)$ be the maximal tree containing node $i$ rooted at node $i$. Suppose that there are two distinct infinite paths $P^{1}=i-i_{1}-i_{2}-\ldots$ and $P^{2}=i-\bar{\imath}_{1}-\bar{l}_{2}-\cdots$ in $T$. Then $i_{1} \in C_{T}(i)$ and $i_{k+1} \in C_{T}\left(i_{k}\right)$ for $k=1,2, \ldots$ Similarly, $\bar{l}_{1} \in C_{T}(i)$ and $\bar{l}_{k+1} \in C_{T}\left(\bar{l}_{k}\right)$ for $k=1,2, \ldots$ We note that every finite cut $S \in \mathbf{S}\left(T\left(i_{1}\right)\right)$ must contain an arc from the path $P^{1}$. Because $A\left(P^{1}\right) \subseteq A(x), r\left(i^{\prime}, j^{\prime}\right) \geq \theta$ for $\left(i^{\prime}, j^{\prime}\right) \in A\left(P^{1}\right)$. Therefore, by definition, $R_{T}\left(i_{1}\right) \geq \theta>0$. Using a similar argument, $R_{T}\left(\bar{l}_{1}\right) \geq \theta>0$. However, this contradicts the assumption that the flow satisfies Extreme Point Condition 3.4( $\left.\mathrm{b}^{\prime}\right)$ because $i_{1}, \bar{l}_{1} \in C_{T}(i)$. Hence, there cannot be two distinct infinite paths starting at node $i$, and the flow $x$ must satisfy Extreme Point Condition 3.8(b).

We note that the proof of Theorem 3.10 shows that Extreme Point Condition 3.8 is sufficient for a feasible flow $x$ to be an extreme point in general. In the special case where the network flow problem satisfies Nonvanishing Support Assumption 3.9 the conditions are necessary as well, as the theorem states. Note that, in contrast to Extreme Point Condition 3.4, Extreme Point Condition 3.8 is dependent only on the graph $G(x)$ and not the actual value of the flow $x$. We use this observation to prove that if the network flow problem satisfies Nonvanishing Support Assumption 3.9 then any extreme point $x$ can be expressed in terms of its restricted arcs.

Theorem 3.11. Suppose that the network flow problem given by $G=(N, A), \ell, u$, and $d$ satisfies Nonvanishing Support Assumption 3.9. Then any extreme point $x$ for the problem is uniquely characterized by the sets $L(x)$ and $U(x)$.

Proof. Suppose that $x^{1}$ and $x^{2}$ are two extreme points such that $L\left(x^{1}\right)=L\left(x^{2}\right), U\left(x^{1}\right)=U\left(x^{2}\right)$, and $x^{1} \neq x^{2}$. Given our observation, $G\left(x^{1}\right)$ and $G\left(x^{2}\right)$ must be identical. Now consider the feasible flow $x=\left(x^{1}+x^{2}\right) / 2$. By Proposition 2.1, $L(x)=L\left(x^{1}\right)=L\left(x^{2}\right)$ and $U(x)=U\left(x^{1}\right)=U\left(x^{2}\right)$. We observe that for any feasible flow $x$, the graph $G(x)$ can be specified by providing the sets $L(x)$ and $U(x)$ because $L(x) \cup U(x) \cup A(x)=A$. This implies that $G(x), G\left(x^{1}\right)$, and $G\left(x^{2}\right)$ are identical. Because $x^{1}$ is an extreme point, $G(x)=G\left(x^{1}\right)$ satisfies the conditions of Theorem 3.10.
However, this leads to a contradiction because Theorem 3.10 then implies that $x$ is an extreme point. Therefore, we cannot have two extreme points $x^{1} \neq x^{2}$ such that $L\left(x^{1}\right)=L\left(x^{2}\right)$ and $U\left(x^{1}\right)=U\left(x^{2}\right)$.

In Section 3.4 we will show that infinite network flow problems where the node demands and arc capacities are integral always have integral valued extreme points. Such problems therefore satisfy Nonvanishing Support Assumption 3.9 with $\theta=1$. This implies that the class of network flow problems satisfying Nonvanishing Support Assumption 3.9 is large and significant because it contains all network flow problems with integral data. We will start by showing that for this class of problems each extreme point can be characterized by partitioning the flow variables into basic variables and nonbasic variables.

3.3.2. Characterization of Extreme Points through Basic and Nonbasic Variables. In the spirit of Extreme Point Condition 3.8, we will define a set of basic arcs or variables as a set $B \subseteq A$ with the following properties:

\section{Condition 3.12.}

(a) The graph $G_{B}=(N, B)$ contains no cycles.

(b) For each node $i \in N$ there exists exactly one infinite path $i-i_{1}-i_{2}-\cdots$ in $G_{B}$.

Now consider a set $B$ satisfying Condition 3.12. Note that this set is maximal in the sense that there does not exist a set $B^{\prime}$ such that $B \subset B^{\prime} \subseteq A$ satisfies Condition 3.12. Moreover, suppose we fix the flow on all $\operatorname{arcs}$ in $A \backslash B$ to an arbitrary value, say $\bar{x}_{i j}$ for $(i, j) \in A \backslash B$. Then it follows immediately that the flows on all other arcs that satisfy the flow balance constraints are uniquely defined. This can be seen as follows. Consider any arc in $(i, j) \in B$. This arc is on exactly one infinite path. Therefore, this infinite path must have a leaf node, because it is, by definition, only infinite in one direction. The value of the arc adjacent to the leaf node can then be found through the flow balance equation for that node. (Note that the value of the flow on this arc may violate the bound constraints, in which case the solution that we are constructing is infeasible.) Now remove the arc from $G_{B}$ and proceed with the reduced network.

In summary, we will call any set $B$ satisfying Condition 3.12 a set of basic variables (and its complement will be the corresponding set of nonbasic variables). By analogy with the concept of basic and nonbasic variables in finite dimensional linear programming, any solution satisfying the flow balance constraints that is found by choosing a basic set $B$ and fixing the corresponding nonbasic variables to either their lower or upper bound is a basic solution. If this solution also satisfies all bound constraints it is a basic feasible solution; otherwise, it is a basic infeasible solution. Note that this immediately implies that any basic feasible solution is an extreme point.

It remains to be shown that for each extreme point solution $x$ there exists an associated set $B(x) \supseteq A(x)$ satisfying 
Condition 3.12. If this is the case, it immediately follows that $x$ is a basic feasible solution. We will show that this is the case when the network flow problem satisfies Nonvanishing Support Assumption 3.9.

Theorem 3.13. Suppose that the network flow problem given by $G=(N, A), \ell, u$, and $d$ satisfies Nonvanishing Support Assumption 3.9. Then any extreme point solution is a basic feasible solution.

Proof. It is clear that an extreme point $x$ is a basic feasible solution if $G(x)$ happens to satisfy Condition 3.12. If it does not, this means that there are some nodes in $N$ that are not in any infinite path; we therefore need to show that we can enlarge $G(x)$ with such a path for all such nodes without creating any cycles or doubly-infinite paths. To this end, we apply the following procedure:

STEP 0. Initialize $B=A(x)$.

STEP 1. Let $G^{\prime}=\left(N^{\prime}, B\right)$ be the graph induced by $B$, that is,

$$
N^{\prime}=\{i \in N: \exists j \in N \text { such that }(i, j) \in B \text { or }(j, i) \in B\}
$$

and let $G^{\prime \prime}=\left(N \backslash N^{\prime}, A^{\prime \prime}\right)$ be the graph induced by $N \backslash N^{\prime}$, i.e.,

$$
A^{\prime \prime}=\left\{(i, j) \in A: i, j \in N \backslash N^{\prime}\right\} .
$$

STEP 2. If $G^{\prime \prime}$ contains an infinite path then identify one, add all its arcs to the set $B$, and return to Step 1. Otherwise, stop.

This procedure produces some set $B$. Note that this set $B$ will contain exactly one infinite path from each node in its node set $N^{\prime}$. Moreover, it will not contain any cycles because it is the union of an acyclic subnetwork and a set of disjoint infinite paths. If $N^{\prime}=N$, that is, $B$ contains an arc adjacent to all nodes in $N$, it follows that $B$ is the basic set corresponding to $x$.

However, suppose that $G^{\prime} \neq G$. Then the corresponding set $G^{\prime \prime}$ must be a countable collection of disjoint finite subgraphs. In that case we perform the following procedure:

STEP 1. Choose one of the finite subgraphs of $G^{\prime \prime}$.

STEP 2. Find a spanning tree $T$ in this subgraph.

STEP 3. Find an arc $(i, j)$ that connects $T$ to $G^{\prime}$ (note that such an arc indeed exists).

STEP 4. Add the arcs in $T$ as well as the connecting arc $(i, j)$ to $B$ and remove the subgraph chosen in Step 1 from $G^{\prime \prime}$.

STEP 5. If $G^{\prime \prime} \neq \varnothing$, return to Step 1 .

This procedure produces a new set $B$. We claim that this set satisfies Condition 3.12. First, observe that the first procedure ensures that an infinite path exists from all nodes in the graph
$G^{\prime}$ that results from this procedure. Furthermore, Step 4 in the second procedure ensures that such a path exists for all other nodes. Therefore, each node in $N$ contains a path to infinity only consisting of arcs in $B$. Second, note that the graph $G^{\prime}$ that results from the first procedure is acyclic and the second procedure connects finite acyclic graphs to this graph. This means that the second procedure cannot create a cycle or a doubly-infinite path. Therefore, the set $B$ does not contain any cycles nor a node that is on more than one infinite path.

\subsection{Integral Network Flow Problems}

In this section, we discuss a class of network flow problems where the demands $d$ and the arc capacities $u$ can only take integral values. It is well known (see, e.g., [1]) that in the case of finite networks, if the node demands and the arc capacities are integral, then any extreme point must have integral values for arc flows. We extend this result to infinite network flow problems.

To prove this result we need to introduce some new notation. For any real number $a$, we define $a^{f}=\min \{a-$ $\lfloor a\rfloor,\lceil a\rceil-a\}$. For a given flow $x \in \mathbb{R}_{+}^{|A|}$, we define its rounding vector $x^{f} \in[0,0.5]^{|A|}$ through $x^{f}(i, j)=(x(i, j))^{f}$ for $(i, j) \in A$.

Theorem 3.14. If the demands $d$ as well as the lower and upper bounds $\ell$ and $u$ in a network flow problem only take integer values, then any extreme point of the problem is integral.

Proof. We will show that any feasible flow $x$ that has a fractional value for some arc in $A$ does not satisfy Extreme Point Condition 3.4, so it cannot be an extreme point. Hence, the extreme points must have integral values for all arcs.

From Lemma 3.2, if the graph $G(x)$ contains a cycle then $x$ cannot be an extreme point. So suppose that we have a feasible flow $x$ such that $G(x)$ contains no cycle and for some $(i, j) \in A(x), x(i, j)$ has a fractional value [i.e., $x^{f}(i, j)>0$ ]. Let $T=\left(N^{\prime}, A^{\prime}, i\right)$ be the maximal tree containing the arc $(i, j)$ rooted at $i$. Because the flow $x$ satisfies the flow balance Equation (1) at node $i$ and $d(i)$ is an integer, there must exist another arc incident to node $i$ [different from $(i, j)]$ that has fractional flow. There are two possible cases:

(1) there is an $\operatorname{arc}(i, \bar{j})$ in $A^{\prime}$ such that $\bar{j} \neq j$ and $x(i, \bar{j})$ is fractional;

(2) there is an $\operatorname{arc}(\bar{\jmath}, i)$ in $A^{\prime}$ such that $\bar{j} \neq j$ and $x(\bar{j}, i)$ is fractional.

We deal with case (1); case (2) can be handled similarly. We observe that if the lower and upper bounds $\ell$ and $u$ only take integer values, then for any feasible flow $x$, $r(i, j) \geq x^{f}(i, j)$ for $\left(i^{\prime}, j^{\prime}\right) \in A$. By definition, $R_{T}(j)=$ $\inf _{S \in \mathbf{S}(T(j))} \sum_{\left(i^{\prime}, j^{\prime}\right) \in S} r\left(i^{\prime}, j^{\prime}\right)$. From our observation it follows that $R_{T}(j) \geq \inf _{S \in \mathbf{S}(T(j))} \sum_{\left(i^{\prime}, j^{\prime}\right) \in S} x^{f}\left(i^{\prime}, j^{\prime}\right)$. We now use Lemma A.3 in the Appendix to conclude that $R_{T}(j) \geq$ $x^{f}(i, j)>0$ as $i=p_{T}(j)$ in $T$. By a similar argument, we can 
conclude that $R_{T}(\bar{J}) \geq x^{f}(i, \bar{J})>0$. This means that node $i$ has two children $j$ and $\bar{j}$ such that $R_{T}(j), R_{T}(\bar{j})>0$, and therefore $x$ does not satisfy Extreme Point Condition 3.4( $\left(\mathrm{b}^{\prime}\right)$, which is equivalent to Extreme Point Condition 3.4(b).

The integrality of the extreme points in case the problem data are integral immediately implies that Nonvanishing Support Assumption 3.9 is satisfied:

Corollary 3.15. If the demands $d$ as well as the lower and upper bounds $\ell$ and $u$ in a network flow problem only take integer values, then Nonvanishing Support Assumption 3.9 is satisfied with $\theta=1$.

In turn, this then implies that Extreme Point Condition 3.8 characterizes all extreme points to network flow problems with integral data.

\section{AN APPLICATION TO THE INFINITE HORIZON CAPACITATED ECONOMIC LOT-SIZING PROBLEM}

In this section we will study the extreme point structure of the infinite horizon variant of the classical capacitated economic lot-sizing problem with backordering. In this problem, the uncapacitated version of which dates back to Wagner and Whitin [16] and Zangwill [17], a producer faces a deterministic demand stream for a single product over a sequence of time periods. This demand needs to be satisfied from production, which may be held in inventory until demand occurs or used to satisfy past demands through backordering. The problem is then to decide how much to produce in each time period to ensure that all demands are satisfied at minimum production, inventory holding, and backordering costs. To formulate this problem as an optimization problem, let us denote the time periods by $t=1,2, \ldots$ and the (integral) demand in time period $t$ by $d_{t}$. Furthermore, let $c_{t}(\cdot), h_{t}^{+}(\cdot)$, and $h_{t}^{-}(\cdot)$ denote the production, inventory holding, and backlogging cost functions in period $t$, and assume that they are concave and nondecreasing, where we assume that inventory and backordering costs are charged against end of period inventory and backorder levels. Let $\bar{p}_{t}, \bar{I}_{t}^{+}$, and $\bar{I}_{t}^{-}$denote the (integral and finite) production, inventory, and backorder limits in period $t$. Finally, denote the quantity produced in period $t$ by $p_{t}$, the quantity in inventory at the end of period $t$ by $I_{t}^{+}$, and the quantity backordered at the end of period $t$ by $I_{t}^{-}$. The problem can then be formulated as:

$$
\operatorname{minimize} \sum_{t=1}^{\infty}\left(c_{t}\left(p_{t}\right)+h_{t}^{+}\left(I_{t}^{+}\right)+h_{t}^{-}\left(I_{t}^{-}\right)\right)
$$

subject to

$$
\begin{array}{rlrl}
p_{1}+I_{1}^{-} & =d_{1}+I_{1}^{+} & & \\
p_{t}+I_{t-1}^{+}+I_{t}^{-} & =d_{t}+I_{t-1}^{-}+I_{t}^{+} & & t=2,3, \ldots \\
p_{t} & \leq \bar{p}_{t} & t & =1,2, \ldots \\
I_{t}^{+} & \leq \bar{I}_{t}^{+} & t & =1,2, \ldots
\end{array}
$$

$$
\begin{array}{rlrl}
I_{t}^{-} & \leq \bar{I}_{t}^{-} & t & =1,2, \ldots \\
p_{t}, I_{t}^{+}, I_{t}^{-} & \geq 0 & t & =1,2, \ldots
\end{array}
$$

When viewed as a subset of the product space $\prod_{i=1}^{\infty} \mathbb{R}^{3}$, the feasible region is clearly compact. Due to the concavity of the cost functions, we then know that if the lot-sizing problem has an optimal solution, an extreme point optimal solution exists by Bauer's Minimum Principle (see [13]). An optimal solution, in turn, exists if there exists a feasible solution with finite cost value. [This is the case if, for example, (i) the cost functions are linear and discounted at a rate in $(0,1)$ and (ii) $d_{t} \leq \bar{p}_{t} \leq \bar{p}<\infty$ for all $t=1,2, \ldots$. It is therefore of interest to study the extreme point structure of the feasible region of the economic lot-sizing problem.

When the horizon is finite, say $T$ periods, the first step is usually to formulate the problem as a minimum cost network flow problem. In the most common network flow formulation (see Fig. 5), each period $t$ is represented by a demand node with demand $d_{t}$, and production is represented by a source node with supply equal to the sum of all demands, that is, the demand of the source node is equal to $-\sum_{t=1}^{T} d_{t}$. Production in period $t$ is represented by a directed arc from the source node to node $t$ with capacity $\bar{p}_{t}$, inventory carried from period $t$ to period $t+1$ is represented by a directed arc from node $t$ to node $t+1$ with capacity $\bar{I}_{t}^{+}$, and backorders carried from period $t+1$ to period $t$ are represented by a directed arc from node $t+1$ to node $t$ with capacity $\bar{I}_{t}^{-}$. In this network, each feasible solution to the lot-sizing problem is represented by a feasible flow.

This network flow formulation of the finite horizon economic lot-sizing problem can be used to derive properties of the extreme point solutions to this problem. It is well known that any extreme point solution satisfies the following conditions (see, e.g., [9]):

(1) All production and inventory quantities are integral valued.

(2) In no period $t=1,2, \ldots$ do we have $0<I_{t}^{+}<\bar{I}_{t}^{+}$and $0<I_{t}^{-}<\bar{I}_{t}^{-}$.

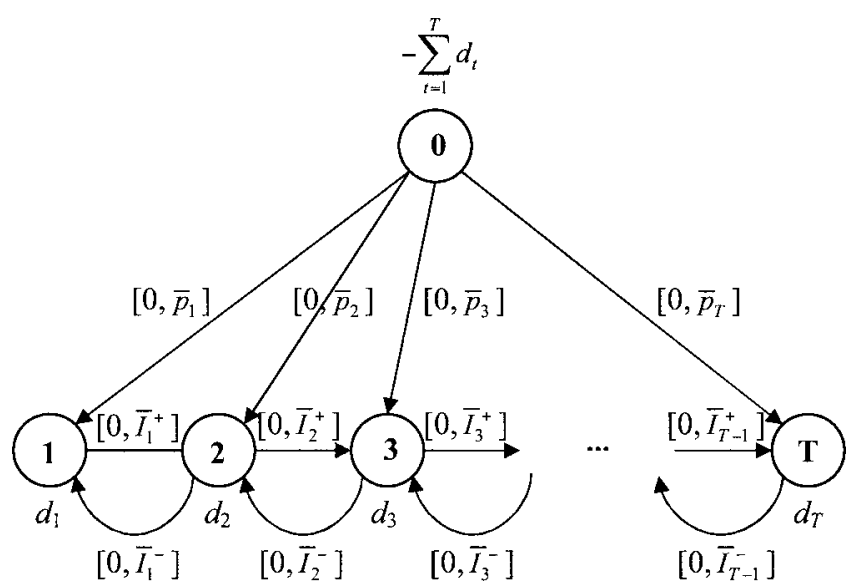

FIG. 5. A network representation of the finite horizon economic lot-sizing problem. 
(3) Consider two periods, say $t_{1}$ and $t_{2}$, in which the inventory and backorder levels are either at zero or their capacity, while $0<I_{t}^{+}<\bar{I}_{t}^{+}$or $0<I_{t}^{-}<\bar{I}_{t}^{-}$for all $t=t_{1}+1, \ldots, t_{2}-1$ (such a sequence is often called a block or subplan). Then at most one of the production quantitites $p_{t}, t=t_{1}+1, \ldots, t_{2}$, satisfies both its upper and lower bound strictly, that is, there exists at most one $t=t_{1}+1, \ldots, t_{2}$ such that $0<p_{t}<\bar{p}_{t}$.

The goal of this section is to generalize these properties to the infinite horizon case. In fact, we will show that, in addition, the following condition holds:

(4) Consider a period, say $t_{1}$, in which the inventory and backorder levels are either at zero or their capacity, while $0<I_{t}^{+}<\bar{I}_{t}^{+}$or $0<I_{t}^{-}<\bar{I}_{t}^{-}$for all $t=t_{1}+1, t_{1}+2, \ldots$ (i.e., such a sequence generalizes the concept of a block or subplan). Then at most one of the production quantitites $p_{t}, t=t_{1}+1, t_{1}+2, \ldots$, satisfies both its upper and lower bound strictly, that is, there exists at most one $t=$ $t_{1}+1, t_{1}+2, \ldots$ such that $0<p_{t}<\bar{p}_{t}$.

When attempting to generalize the network flow approach to the infinite horizon economic lot-sizing problem, it is immediate that we cannot use a network of the same form as in the finite horizon case. Because all demands are integral, total demand will be infinite if we are dealing with a truly infinite horizon problem. This implies that the total production over the infinite horizon should be infinite as well. However, this means that the supply of the source node is not well defined in the straightforward generalization of the network. Another complication is that the total out degree of the source node in this generalization would be infinite, while the results on the structure of extreme points of infinite network flow problems derived in this article require that the in and out degrees of all nodes in the network are finite. We therefore consider a new network flow formulation of the problem. In this formulation, each period is still represented by a demand node, and inventory and backorder flows are represented by capacitated directed arcs between successive period nodes. However, production in each period $t$ is represented by an infinite directed path leading to period $t$ 's node. All nodes on this infinite path have demand 0 , all arcs have capacity $\bar{p}_{t}$, and all arcs have cost zero except for the last arc, that is, the arc leading from node $(t, 1)$ to node $t$ (see Fig. 6 for an illustration of this network). This representation of the problem eliminates the need for specifying the total production in advance, because there is no node that acts as a source. Instead, the infinite paths in each period $t$ ensure that any production quantity not exceeding the capacity can be produced.

All results regarding the extreme point structure of infinite horizon network flow problems that have been derived in this article now apply to this representation of the infinite horizon economic lot-sizing problem. Theorem 3.14 now immediately generalizes extreme point property (1) stated above to the infinite dimensional case. But Corollary 3.15 also states that, because the demands are integral, we know that Extreme Point Condition 3.8 characterizes the extreme points to this problem. Recall that this condition says that, for any extremal flow, say $x$, we have

(a) $G(x)$ contains no cycles;

(b) for any node $i$ there exists at most one infinite path $i-$ $i_{1}-i_{2}-\cdots$ in $G(x)$.

Extreme point property (2) now follows immediately from condition (a). Moreover, we can immediately conclude that any feasible solution $x$ satisfying property (2) corresponds to an acyclic free arc graph $G(x)$. But it is also easy to see that not all such feasible flows are extreme point solutions. For instance, if all but a finite number of the demands are equal to zero, we essentially have a finite lot-sizing problem, and clearly not all feasible solutions to such a problem that satisfy property (2) are extreme points. However, condition (b) allows us to generalize property (3) to the infinite dimensional case. This can be seen as follows. Consider a feasible
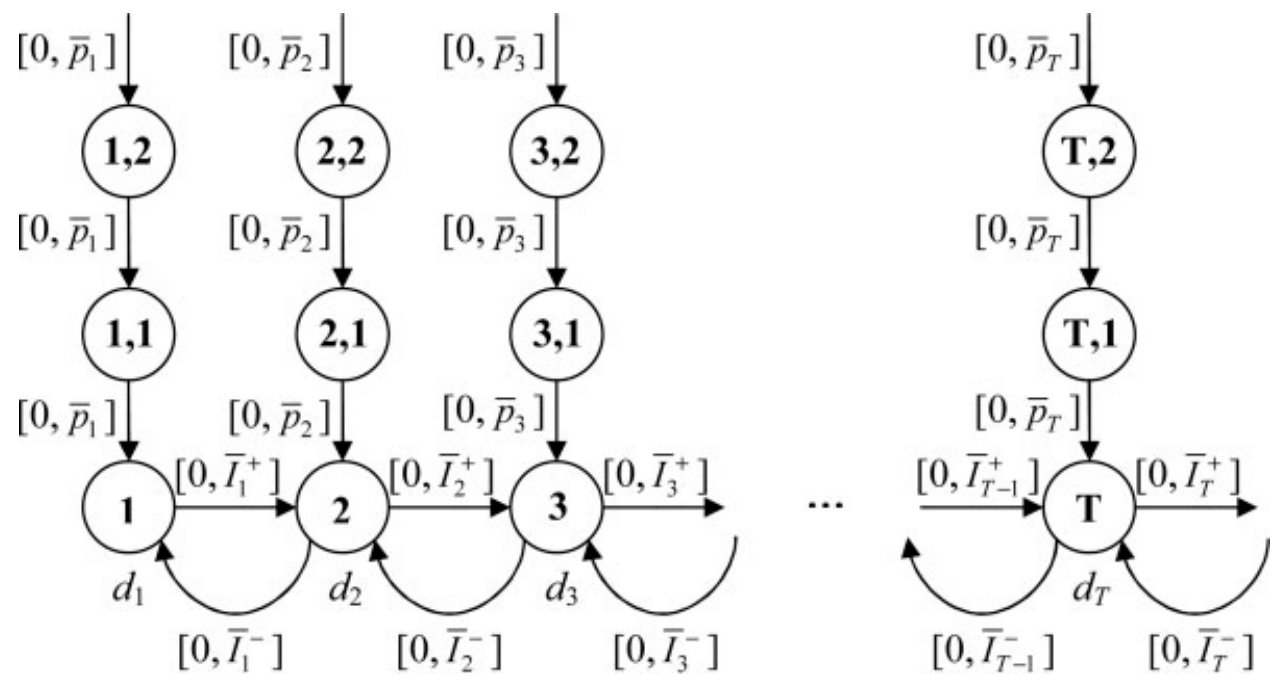

FIG. 6. A network representation of the infinite horizon economic lot-sizing problem. 
solution and let $t_{1}$ and $t_{2}$ identify a block. Now suppose that $0<p_{t}<\bar{p}_{t}$ for two values of $t=t_{1}+1, \ldots, t_{2}$, say $s_{1}$ and $s_{2}$. This means that node $s_{1}$ is on two infinite paths in $G(x)$ : $\cdots-\left(s_{1}, 2\right)-\left(s_{1}, 1\right)-s_{1}$ and $s_{1}-\cdots-s_{2}-\left(s_{2}, 1\right)-\left(s_{2}, 2\right)-$ $\cdots$, contradicting Extreme Point Condition 3.8(b). Therefore, the solution cannot be an extreme point. In addition, condition (b) allows us to derive property (4) for the infinite dimensional case. Similar to the earlier property, consider a feasible solution and let $t_{1}+1, t_{1}+2, \ldots$ be a (generalized) block. Now suppose that $0<p_{t}<\bar{p}_{t}$ for two values of $t=t_{1}+1, t_{1}+2, \ldots$, say $s_{1}$ and $s_{2}$. This again means that node $s_{1}$ is on two infinite paths in $G(x): \cdots-\left(s_{1}, 2\right)-\left(s_{1}, 1\right)-s_{1}$ and $s_{1}-\cdots-s_{2}-\left(s_{2}, 1\right)-\left(s_{2}, 2\right)-\cdots$, contradicting Extreme Point Condition 3.8(b).

Finally, we would like to remark that a similar approach to the one in this section may be used to show that any extreme point to the uncapacitated infinite horizon economic lotsizing problem without backordering satisfies the standard zero-inventory ordering property that production can only take place when there is no inventory, that is, $I_{t-1} x_{t}=0$ for all $t=1,2, \ldots$.

\section{CONCLUSIONS AND FUTURE RESEARCH}

In this article, we studied the structure of the extreme points of infinite network flow problems. Our results show that, in contrast with the finite dimensional case, an extreme point for an infinite network flow problem can, in general, not be uniquely represented in terms of free arcs. Nevertheless, we developed necessary and sufficient conditions for a feasible flow to be an extreme point that generalize this result. Moreover, under a regularity condition that is met by network flow problems with integral data, we show that an extreme point can be uniquely characterized by a set of free arcs corresponding to a subnetwork containing no finite or infinite cycles. Finally, we showed that, when all problem data are integral, the extreme points always have integral values, extending a result for finite network flow problems to the infinite case.

In future research we hope to generalize our structural results on extreme points and basic feasible solutions to infinite dimensional network flow problems to larger classes of doubly-infinite linear programming problems. Moreover, we intend to use the results of this article and their extensions to develop generalizations of the network simplex method for solving linear minimum cost infinite network flow problems and the simplex method for solving doubly-infinite linear programming problems.

\section{APPENDIX}

Lemma 3.3. Given a rooted tree $T=\left(N^{\prime}, A^{\prime}, r\right)$, the function $R_{T}(i)$ for $i \in N^{\prime}$ is given by

$$
R_{T}(i)=\sum_{j \in C_{T}(i)} \min \left\{\alpha_{i j}, R_{T}(j)\right\}
$$

where

$$
\alpha_{i j}= \begin{cases}r(i, j) & \text { if }(i, j) \in A^{\prime} \\ r(j, i) & \text { otherwise }\end{cases}
$$

Proof. We observe that a finite cut $S \in \mathbf{S}(T(i))$ can be partitioned into $\left|C_{T}(i)\right|$ disjoint sets $S^{j}\left(j \in C_{T}(i)\right)$ where $S^{j}$ either contains a finite cut of the subtree $T(j)$ or contains the arc between node $j$ and $i$ in $T$. Conversely, if we choose sets $S^{j}$ for $j \in C_{T}(i)$ such that $S^{j}$ is either a finite cut of subtree $T(j)$ or contains only the arc between node $j$ and node $i$, then $\cup_{j \in C_{T}(i)} S^{j}$ is a finite cut. Therefore, we can write

$\mathbf{S}(T(i))=\left\{\bigcup_{j \in C_{T}(i)} S^{j}: S^{j} \in \mathbf{S}(T(j))\right.$ or $S^{j}$ is the arc between $i$ and $\left.j\right\}$.

If the arc between $i$ and $j$ is $(i, j)$, the lemma now follows from this observation because

$$
\begin{aligned}
R_{T}(i) & =\inf _{S \in \mathbf{S}(T(i))} \sum_{\left(i^{\prime}, j^{\prime}\right) \in S} r\left(i^{\prime}, j^{\prime}\right) \\
& =\inf _{S \in\left\{\cup_{j \in C_{T}\left(i^{i}\right)} S^{\prime}: S^{j} \in \mathbf{S}(T(j)) \cup\{(i, j)\}\right\}} \sum_{\left(i^{\prime} j^{\prime}\right) \in S} r\left(i^{\prime}, j^{\prime}\right) \\
& =\sum_{j \in C_{T}(i)} \inf _{S^{j} \in \mathbf{S}(T(j)) \cup\{(i, j)\}} \sum_{\left(i^{\prime}, j^{\prime}\right) \in S^{j}} r\left(i^{\prime}, j^{\prime}\right) \\
& =\sum_{j \in C_{T}(i)} \min \left\{r(i, j), \inf _{S^{j} \in \mathbf{S}(T(j))} \sum_{\left(i^{\prime}, j^{\prime}\right) \in S^{j}} r\left(i^{\prime}, j^{\prime}\right)\right\} \\
& =\sum_{j \in C_{T}(i)} \min \left\{\alpha_{i j}, R_{T}(j)\right\} .
\end{aligned}
$$

The case where the arc between $i$ and $j$ is $(j, i)$ follows in an analogous fashion.

The following lemma formally proves the equivalence of Extreme Point Conditions 3.4(b) and 3.4( $\left.\mathrm{b}^{\prime}\right)$. This equivalence result is used to establish the necessity and sufficiency of Extreme Point Condition 3.4 for any feasible flow to be an extreme point.

Lemma A.1. If a feasible flow $x$ satisfies Extreme Point Condition 3.4(b) then it also satisfies Extreme Point Condition $3.4\left(b^{\prime}\right)$.

Proof. Consider a rooted maximal tree $T=\left(N^{\prime}, A^{\prime}, r\right)$ in $G(x)$ such that Extreme Point Condition 3.4(b) is satisfied. We first show that Extreme Point Condition 3.4(b) is also satisfied for $T^{\prime}=\left(N^{\prime}, A^{\prime}, r^{\prime}\right)$ where $r^{\prime} \in C_{T}(r)$, and then generalize the result to any choice of root node in $N^{\prime}$, which proves the lemma. 
Note that when the root for $T$ is changed from $r$ to $r^{\prime} \in C_{T}(r)$, the parent-child relationship is modified only for the nodes $r$ and $r^{\prime}$. That is, for each node $i \in N^{\prime} \backslash\left\{r, r^{\prime}\right\}$ we have $C_{T}(i)=C_{T^{\prime}}(i)$. By Lemma 3.3 we then conclude that $R_{T}(i)=R_{T^{\prime}}(i)$ for any node $i \in N^{\prime} \backslash\left\{r, r^{\prime}\right\}$. Therefore, for all nodes $i \in N^{\prime} \backslash\left\{r, r^{\prime}\right\}$ there exists at most one $j \in C_{T^{\prime}}(i)$ such that $R_{T^{\prime}}(j)>0$. Next, consider node $r$. Note that $r^{\prime}$ is a child of $r$ in $T$ but not in $T^{\prime}$, so that $C_{T^{\prime}}(r)=C_{T}(r) \backslash\left\{r^{\prime}\right\}$. Because $R_{T}(i)=R_{T^{\prime}}(i)$ for all $i \in C_{T^{\prime}}(r) \subset C_{T}(r)$, node $r$ has at most one child $j \in C_{T^{\prime}}(r)$ such that $R_{T^{\prime}}(j)>0$. Finally, consider node $r^{\prime}$. Note that $r$ is a child of $r^{\prime}$ in $T^{\prime}$ but not in $T$, so that $C_{T^{\prime}}\left(r^{\prime}\right)=C_{T}\left(r^{\prime}\right) \cup\{r\}$. Because Extreme Point Condition 3.4(b) is satisfied for $T$, if there exists some $j \in C_{T}(r) \backslash\left\{r^{\prime}\right\}$ with $R_{T}(j)>0$ then $R_{T}\left(r^{\prime}\right)=0$. In that case, Lemma 3.3 shows that all $j \in C_{T}\left(r^{\prime}\right)$ must have $R_{T}(j)=0$ and therefore $R_{T^{\prime}}(j)=0$ for all $j \in C_{T^{\prime}}\left(r^{\prime}\right) \backslash\{r\}$. On the other hand, if $R_{T}\left(r^{\prime}\right)>0$ then $R_{T}(j)=0$ for all $j \in C_{T}(r) \backslash\left\{r^{\prime}\right\}$. In that case, $R_{T^{\prime}}(j)=R_{T}(j)=0$ for all $j \in C_{T^{\prime}}(r)=$ $C_{T}(r) \backslash\left\{r^{\prime}\right\}$ so that Lemma 3.3 implies that $R_{T^{\prime}}(r)=0$. Therefore, because $R_{T^{\prime}}(j)=R_{T}(j)$ for all $j \in C_{T^{\prime}}\left(r^{\prime}\right) \backslash\{r\}$ there exists at most one $j \in C_{T^{\prime}}\left(r^{\prime}\right)$ such that $R_{T^{\prime}}(j)>0$. Hence, Extreme Point Condition 3.4(b) is satisfied for $T^{\prime}$.

Now consider an arbitrary node $r^{\prime} \in N^{\prime}$, and let again $T^{\prime}=\left(N^{\prime}, A^{\prime}, r^{\prime}\right)$. Let $r=i_{1}, \ldots, i_{k}=r^{\prime}$ be the nodes on the unique finite path between $r$ and $r^{\prime}$ in $T$ for some $k \geq 2$. Because $i_{1}$ is a child of $r$ in $T$ rooted at $r$ we can apply the result above to conclude that Extreme Point Condition 3.4(b) is satisfied for $T^{\prime \prime}=\left(N^{\prime}, A^{\prime}, i_{1}\right)$. By repeatedly applying the earlier result in this proof a finite number of times we conclude that Extreme Point Condition 3.4(b) is satisfied for $T^{\prime}$.

The next lemma is a direct consequence of the flow balance equations satisfied by feasible flows.

Lemma A.2. Let $x$ and $x^{\prime}$ be two feasible flows such that $L(x) \subseteq L\left(x^{\prime}\right), U(x) \subseteq U\left(x^{\prime}\right)$, and $G(x)$ contains no cycles. Let $T=\left(N^{\prime}, A^{\prime}, r\right)$ be a maximal tree in $G(x)$ rooted at node $r \in N^{\prime}$. Then, for any node $i \in N^{\prime}$ :

- if $\left(i, p_{T}(i)\right) \in A^{\prime}$ then $\left|x\left(i, p_{T}(i)\right)-x^{\prime}\left(i, p_{T}(i)\right)\right| \leq$ $\sum_{\left(i^{\prime}, j^{\prime}\right) \in S}\left|x\left(i^{\prime}, j^{\prime}\right)-x^{\prime}\left(i^{\prime}, j^{\prime}\right)\right|$ for all $S \in \mathbf{S}(T(i))$

- if $\left(p_{T}(i), i\right) \in A^{\prime}$ then $\left|x\left(p_{T}(i), i\right)-x^{\prime}\left(p_{T}(i), i\right)\right| \leq$ $\sum_{\left(i^{\prime}, j^{\prime}\right) \in S}\left|x\left(i^{\prime}, j^{\prime}\right)-x^{\prime}\left(i^{\prime}, j^{\prime}\right)\right|$ for all $S \in \mathbf{S}(T(i))$.

Proof. We deal explicitly with the case $\left(i, p_{T}(i)\right) \in A^{\prime}$; the result for the other case can be proven in a similar manner. Consider a node $i \in N^{\prime}$ and some $S \in \mathbf{S}(T(i))$ in the rooted tree $T$. Let $\bar{N}$ be the finite set of nodes in $T(i)$ that are connected to node $i$ after the edges in $S$ are removed from $T(i)$. Because $L(x) \subseteq L\left(x^{\prime}\right)$ and $U(x) \subseteq U\left(x^{\prime}\right)$, the flows $x$ and $x^{\prime}$ can only differ over the edges in $A(x)$. Further, because both the flows satisfy the flow balance Equation (1) over the nodes in $\bar{N}$, the difference between the total flow on the arcs from set $N^{\prime} \backslash \bar{N}$ to $\bar{N}$ under $x$ and $x^{\prime}$ should be equal to the difference between the total flow on the $\operatorname{arcs}$ from $\bar{N}$ to $N^{\prime} \backslash \bar{N}$ under $x$ and $x^{\prime}$. That is,

$$
\begin{aligned}
\sum_{\left(i^{\prime}, j^{\prime}\right) \in A(x): i^{\prime} \in \bar{N}, j^{\prime} \in N \backslash \bar{N}}\left(x\left(i^{\prime}, j^{\prime}\right)-x^{\prime}\left(i^{\prime}, j^{\prime}\right)\right) \\
=\sum_{\left(i^{\prime}, j^{\prime}\right) \in A(x): i^{\prime} \in N \backslash \bar{N}, j^{\prime} \in \bar{N}}\left(x\left(i^{\prime}, j^{\prime}\right)-x^{\prime}\left(i^{\prime}, j^{\prime}\right)\right) .
\end{aligned}
$$

Because the set $\bar{N}$ is in the tree $T(i)=\left(N_{T}(i), A_{T}(i)\right)$, the only nodes in $N \backslash \bar{N}$ that are connected to $\bar{N}$ in $A(x)$ are $p_{T}(i)$ and some nodes in $N^{i} \backslash \bar{N}$. Let

$$
\begin{aligned}
& S^{1}=\left\{\left(i^{\prime}, j^{\prime}\right) \in A_{T}(i): i^{\prime} \in \bar{N}, j^{\prime} \in N_{T}(i) \backslash \bar{N}\right\} \\
& S^{2}=\left\{\left(i^{\prime}, j^{\prime}\right) \in A_{T}(i): i^{\prime} \in N_{T}(i) \backslash \bar{N}, j^{\prime} \in \bar{N}\right\} .
\end{aligned}
$$

By our observations,

$$
\begin{aligned}
& \left\{\left(i^{\prime}, j^{\prime}\right) \in A(x): i^{\prime} \in \bar{N}, j^{\prime} \in N \backslash \bar{N}\right\}=\left\{\left(i, p_{T}(i)\right)\right\} \cup S^{1} \\
& \left\{\left(i^{\prime}, j^{\prime}\right) \in A(x): i^{\prime} \in N \backslash \bar{N}, j^{\prime} \in \bar{N}\right\}=S^{2} .
\end{aligned}
$$

Using this information in the equation above,

$$
\begin{aligned}
x\left(i, p_{T}(i)\right)-x^{\prime}\left(i, p_{T}(i)\right)= & \sum_{\left(i^{\prime}, j^{\prime}\right) \in S^{2}}\left(x\left(i^{\prime}, j^{\prime}\right)-x^{\prime}\left(i^{\prime}, j^{\prime}\right)\right) \\
& -\sum_{\left(i^{\prime}, j^{\prime}\right) \in S^{1}}\left(x\left(i^{\prime}, j^{\prime}\right)-x^{\prime}\left(i^{\prime}, j^{\prime}\right)\right) .
\end{aligned}
$$

It follows immediately that

$$
\left|x\left(i, p_{T}(i)\right)-x^{\prime}\left(i, p_{T}(i)\right)\right| \leq \sum_{\left(i^{\prime}, j^{\prime}\right) \in S^{1} \cup S^{2}}\left|\left(x\left(i^{\prime}, j^{\prime}\right)-x^{\prime}\left(i^{\prime}, j^{\prime}\right)\right)\right| .
$$

Finally, we note that by the definition of the set $\bar{N}$ and finite cuts, $S^{1} \cup S^{2} \subseteq S$, and the result follows.

The following result regarding the rounding vector of a feasible flow is used to prove that all extreme points in network flow problems with integral data are integral.

Lemma A.3. Let $x$ be a feasible flow such that $G(x)$ contains no cycles. Let $T=\left(N^{\prime}, A^{\prime}, r\right)$ be a maximal rooted tree in $G(x)$. Then, for any node $i \in N^{\prime}$ :

- if $\left(i, p_{T}(i)\right) \in A^{\prime}$ then $x^{f}\left(i, p_{T}(i)\right) \leq \sum_{\left(i^{\prime}, j^{\prime}\right) \in S} x^{f}\left(i^{\prime}, j^{\prime}\right)$ for all $S \in \mathbf{S}(T(i))$

- if $\left(p_{T}(i), i\right) \in A^{\prime}$ then $x^{f}\left(p_{T}(i), i\right) \leq \sum_{\left(i^{\prime}, j^{\prime}\right) \in S} x^{f}\left(i^{\prime}, j^{\prime}\right)$ for all $S \in \mathbf{S}(T(i))$.

Proof. The proof of this result is similar to the proof of Lemma A.2 in this Appendix. We show the result when $\left(i, p_{T}(i)\right) \in A^{\prime}$; the other case can be shown in an analogous manner. Consider a node $i \in N^{\prime}$ and some $S \in \mathbf{S}(T(i))$ in the rooted tree $T$. Let $\bar{N}$ be the finite set of nodes in $T^{i}=$ $\left(N_{T}(i), A_{T}(i)\right)$ that are connected to node $i$ after the edges 
in $S$ are removed from $T(i)$. Summing up the flow balance equations for the nodes in set $\bar{N}$,

$$
\begin{aligned}
\sum_{\left(i^{\prime}, j^{\prime}\right) \in A(x): i^{\prime} \in N^{\prime} \backslash \bar{N}, j^{\prime} \in \bar{N}} x\left(i^{\prime}, j^{\prime}\right) \\
-\sum_{\left(i^{\prime}, j^{\prime}\right) \in A(x): i^{\prime} \in \bar{N}, j^{\prime} \in N^{\prime} \backslash \bar{N}} x\left(i^{\prime}, j^{\prime}\right)=\sum_{i^{\prime} \in \bar{N}} d\left(i^{\prime}\right) .
\end{aligned}
$$

Because the set $\bar{N}$ is in the tree $T(i)$, the only nodes in $N \backslash \bar{N}$ that are connected to $\bar{N}$ in $A(x)$ are $p(i)$ and some nodes in $N_{T}(i) \backslash \bar{N}$. Let

$$
\begin{aligned}
& S^{1}=\left\{\left(i^{\prime}, j^{\prime}\right) \in A_{T}(i): i^{\prime} \in \bar{N}, j^{\prime} \in N_{T}(i) \backslash \bar{N}\right\} \\
& S^{2}=\left\{\left(i^{\prime}, j^{\prime}\right) \in A_{T}(i): i^{\prime} \in N_{T}(i) \backslash \bar{N}, j^{\prime} \in \bar{N}\right\} .
\end{aligned}
$$

By our observations,

$$
\begin{aligned}
& \left\{\left(i^{\prime}, j^{\prime}\right) \in A(x): i^{\prime} \in \bar{N}, j^{\prime} \in N \backslash \bar{N}\right\}=\left\{\left(i, p_{T}(i)\right)\right\} \cup S^{1} \\
& \left\{\left(i^{\prime}, j^{\prime}\right) \in A(x): i^{\prime} \in N \backslash \bar{N}, j^{\prime} \in \bar{N}\right\}=S^{2} .
\end{aligned}
$$

Using this information in the equation above,

$$
x\left(i, p_{T}(i)\right)=\sum_{i^{\prime} \in \bar{N}} d\left(i^{\prime}\right)+\sum_{\left(i^{\prime} j^{\prime}\right) \in S^{1}} x\left(i^{\prime}, j^{\prime}\right)-\sum_{\left(i^{\prime} j^{\prime}\right) \in S^{2}} x\left(i^{\prime}, j^{\prime}\right) .
$$

Because $\sum_{i^{\prime} \in \bar{N}} d\left(i^{\prime}\right)=0$, it is easy to see that

$$
x^{f}\left(i, p_{T}(i)\right) \leq \sum_{\left(i^{\prime}, j^{\prime}\right) \in S^{1} \cup S^{2}} x^{f}\left(i^{\prime}, j^{\prime}\right) .
$$

Finally, we note that by the definition of the set $\bar{N}$ and finite cuts, $S^{1} \cup S^{2} \subseteq S$, and the result follows.

\section{REFERENCES}

[1] R.K. Ahuja, T.L. Magnanti, and J.B. Orlin, Network flows: Theory, algorithms, and applications, Prentice-Hall, Englewood Cliffs, NJ, 1993.
[2] C.D. Aliprantis and K.C. Border, Infinite dimensional analysis: A hitchhiker's guide, Springer-Verlag, Heidelberg, Germany, 1994.

[3] E.J. Anderson and P. Nash, Linear programming in infinite dimensional spaces, Wiley, New York, 1987.

[4] E.J. Anderson and A.B. Philpott, A continuous-time network simplex algorithm, Networks 19 (1989), 394-425.

[5] J.C. Bean, J.R. Lohmann, and R.L. Smith, A dynamic infinite horizon replacement economy decision model, Eng Economist 30 (1985), 99-120.

[6] J.C. Bean and R.L. Smith, Conditions for the existence of planning horizons, Math Oper Res 9 (1984), 391-401.

[7] J.C. Bean and R.L. Smith, Optimal capacity expansion over an infinite horizon, Manage Sci 31 (1985), 1523-1532.

[8] W.P. Cross, H.E. Romeijn, and R.L. Smith, Approximating extreme points in infinite dimensional convex sets, Math Oper Res 23 (1994), 433-442.

[9] E.V. Denardo, Dynamic programming: Models and applications, Prentice-Hall, Englewood Cliffs, NJ, 1982.

[10] B. Fuchssteiner and K. Morisse, Infinite networks: Minimal cost flows, Appl Math Lett 9 (1996), 89-93.

[11] R. Gomory and T.C. Hu, "Flows in continua," Integer programs and network flows, T.C. Hu (Editor), Addison Wesley, Reading, MA, 1969.

[12] P. Jones, J. Zydiak, and W. Hopp, Stationary dual prices and depreciation, Math Program 41 (1988), 357-366.

[13] N.M. Roy, Extreme points of convex sets in infinite dimensional spaces, Am Math Monthly 94 (1987), 409-422.

[14] I.E. Schochetman and R.L. Smith, Infinite horizon optimization, Math Oper Res 14 (1989), 559-574.

[15] R.L. Smith and R.Q. Zhang, Infinite horizon production planning in time varying systems with convex production and inventory costs, Manage Sci 44 (1998), 1313-1320.

[16] H.M. Wagner and T.M. Whitin, Dynamic version of the economic lot size model, Manage Sci 5 (1958), 89-96.

[17] W.I. Zangwill, A backlogging model and a multi-echelon model of a dynamic economic lot sizing problem, Manage Sci 15 (1969), 506-527. 